Stud. Univ. Babeş-Bolyai Math. 62(2017), No. 2, 233-248

DOI: 10.24193/subbmath.2017.2.09

\title{
Properties of $m$-complex symmetric operators
}

\author{
Muneo Chō, Eungil Ko and Ji Eun Lee
}

Dedicated to the memory of Professor Takayuki Furuta in deep sorrow

\begin{abstract}
In this paper, we study several properties of $m$-complex symmetric operators. In particular, we prove that if $T \in \mathcal{L}(\mathcal{H})$ is an $m$-complex symmetric operator and $N$ is a nilpotent operator of order $n>2$ with $T N=N T$, then $T+N$ is a $(2 n+m-2)$-complex symmetric operator. Moreover, we investigate the decomposability of $T+A$ and $T A$ where $T$ is an $m$-complex symmetric operator and $A$ is an algebraic operator. Finally, we provide various spectral relations of such operators. As some applications of these results, we discuss Weyl type theorems for such operators.
\end{abstract}

Mathematics Subject Classification (2010): 47A11, 47B25.

Keywords: Conjugation, $m$-complex symmetric operator, nilpotent perturbations, decomposable, Weyl type theorems.

\section{Introduction}

Let $\mathcal{L}(\mathcal{H})$ be the algebra of all bounded linear operators on a separable complex Hilbert space $\mathcal{H}$. A conjugation on $\mathcal{H}$ is an antilinear operator $C: \mathcal{H} \rightarrow \mathcal{H}$ which satisfies $\langle C x, C y\rangle=\langle y, x\rangle$ for all $x, y \in \mathcal{H}$ and $C^{2}=I$. For any conjugation $C$, there is an orthonormal basis $\left\{e_{n}\right\}_{n=0}^{\infty}$ for $\mathcal{H}$ such that $C e_{n}=e_{n}$ for all $n$ (see [14] for more details). An operator $T \in \mathcal{L}(\mathcal{H})$ is said to be complex symmetric if there exists a conjugation $C$ on $\mathcal{H}$ such that $T=C T^{*} C$.

This research was supported by Basic Science Research Program through the National Research Foundation of Korea(NRF) funded by the Ministry of Education(2009-0093827). The third author was supported by Basic Science Research Program through the National Research Foundation of Korea(NRF) funded by the Ministry of Education, Science and Technology(2016R1A2B4007035) and this research is partially supported by Grant-in-Aid Scientific Research No.15K04910. 
In 1970, J. W. Helton [18] initiated the study of operators $T \in \mathcal{L}(\mathcal{H})$ which satisfy an identity of the following form;

$$
\sum_{j=0}^{m}(-1)^{m-j}\left(\begin{array}{c}
m \\
j
\end{array}\right) T^{* j} T^{m-j}=0 .
$$

In the light of complex symmetric operators, using the identity (1.1), we define $m$-complex symmetric operators as follows; an operator $T \in \mathcal{L}(\mathcal{H})$ is said to be an $m$-complex symmetric operator if there exists some conjugation $C$ such that

$$
\sum_{j=0}^{m}(-1)^{m-j}\left(\begin{array}{c}
m \\
j
\end{array}\right) T^{* j} C T^{m-j} C=0
$$

for some positive integer $m$. In this case, we say that $T$ is an $m$-complex symmetric operator with conjugation $C$. In particular, if $m=1, T$ is called a 1-complex symmetric operator (simply a complex symmetric operator). The authors have studied spectral properties and local spectral properties of $m$-complex symmetric operators. In particular, they have shown that if $T$ is an $m$-complex symmetric operator with the conjugation $C$, then $T$ is decomposable if and only if $T^{*}$ has the property $(\beta)$ (see $[9])$. Set $\Delta_{m}(T):=\sum_{j=0}^{m}(-1)^{m-j}\left(\begin{array}{c}m \\ j\end{array}\right) T^{* j} C T^{m-j} C$. Then $T$ is an $m$-complex symmetric operator with conjugation $C$ if and only if $\Delta_{m}(T)=0$. An operator $T \in \mathcal{L}(\mathcal{H})$ is said to be a strict $m$-complex symmetric operator if $T$ is an $m$-complex symmetric operator but it is not an $(m-1)$-complex symmetric operator. Note that

$$
T^{*} \Delta_{m}(T)-\Delta_{m}(T)(C T C)=\Delta_{m+1}(T) .
$$

Hence, if $T$ is an $m$-complex symmetric operator with conjugation $C$, then $T$ is an $n$ complex symmetric operator with conjugation $C$ for all $n \geq m$. In sequel, it was shown from [10] that if $m$ is even, then $\Delta_{m}(T)$ is complex symmetric with the conjugation $C$, and if $m$ is odd, then $\Delta_{m}(T)$ is skew complex symmetric with the conjugation $C$. Moreover, we investigate conditions for $(m+1)$-complex symmetric operators to be $m$-complex symmetric operators and characterize the spectrum of $\Delta_{m}(T)$. All normal operators, algebraic operators of order 2, Hankel matrices, finite Toeplitz matrices, all truncated Toeplitz operators, some Volterra integration operators, nilpotent operators of order $k$, and nilpotent perturbations of Hermitian operators are included in the class of $m$-complex symmetric operators (see [14], [15], [16], [19], and [9] for more details). The class of $m$-complex symmetric operators is surprisingly large class.

Many authors have studied Hermitian, isometric, unitary, and normal operators perturbed by nilpotent operators (see [2], [6], [8], and [21], etc). In 2014, T. Bermudez, A. Martinon, V. Muller, and J. Noda ([6]) have been studied the perturbation of $m$ isometries by nilpotent operators. In light of $m$-complex symmetric operators, we consider the nilpotent perturbations of $m$-complex symmetric operators. In particular, we prove that if $T \in \mathcal{L}(\mathcal{H})$ is an $m$-complex symmetric operator and $N$ is a nilpotent operator of order $n>2$ with $T N=N T$, then $T+N$ is a $(2 n+m-2)$ complex symmetric operator. Moreover, we investigate the decomposability of $T+A$ 
or $T A$ where $T$ is $m$-complex symmetric operators. Finally, we provide various spectral relations of such operators. As some applications of these results, we focus on Weyl type theorems for such operators.

\section{Preliminaries}

If $T \in \mathcal{L}(\mathcal{H})$, we write $\sigma(T), \sigma_{s u}(T), \Gamma(T), \sigma_{p}(T), \sigma_{a p}(T), \sigma_{e}(T), \sigma_{l e}(T), \sigma_{r e}(T)$, $\sigma_{b}(T), \sigma_{w}(T), \sigma_{s e}(T)$, and $\sigma_{e s}(T)$ for the spectrum, the surjective spectrum, the compression spectrum, the point spectrum, the approximate point spectrum, the essential spectrum, the left essential spectrum, the right essential spectrum, Browder spectrum, Weyl spectrum, the semi-regular spectrum, and the essentially semi-regular spectrum of $T$, respectively.

An operator $T \in \mathcal{L}(H)$ is said to have the single-valued extension property (or SVEP) if for every open subset $G$ of $\mathbb{C}$ and any $\mathcal{H}$-valued analytic function $f$ on $G$ such that $(T-\lambda) f(\lambda) \equiv 0$ on $G$, we have $f(\lambda) \equiv 0$ on $G$. For an operator $T \in \mathcal{L}(\mathcal{H})$ and for a vector $x \in \mathcal{H}$, the local resolvent set $\rho_{T}(x)$ of $T$ at $x$ is defined as the union of every open subset $G$ of $\mathbb{C}$ on which there is an analytic function $f: G \rightarrow \mathcal{H}$ such that $(T-\lambda) f(\lambda) \equiv x$ on $G$. The local spectrum of $T$ at $x$ is given by $\sigma_{T}(x)=\mathbb{C} \backslash \rho_{T}(x)$. We define the local spectral subspace of $T \in \mathcal{L}(\mathcal{H})$ by $H_{T}(F)=\left\{x \in \mathcal{H}: \sigma_{T}(x) \subset F\right\}$ for a subset $F$ of $\mathbb{C}$. An operator $T \in \mathcal{L}(\mathcal{H})$ is said to have Dunford's property $(C)$ if $H_{T}(F)$ is closed for each closed subset $F$ of $\mathbb{C}$. An operator $T \in \mathcal{L}(\mathcal{H})$ is said to have Bishop's property $(\beta)$ if for every open subset $G$ of $\mathbb{C}$ and every sequence $\left\{f_{n}\right\}$ of $\mathcal{H}$-valued analytic functions on $G$ such that $(T-\lambda) f_{n}(\lambda)$ converges uniformly to 0 in norm on compact subsets of $G$, we get that $f_{n}(\lambda)$ converges uniformly to 0 in norm on compact subsets of $G$. An operator $T \in \mathcal{L}(\mathcal{H})$ is said to be decomposable if for every open cover $\{U, V\}$ of $\mathbb{C}$ there are $T$-invariant subspaces $\mathcal{X}$ and $\mathcal{Y}$ such that

$$
\mathcal{H}=\mathcal{X}+\mathcal{Y}, \sigma\left(\left.T\right|_{\mathcal{X}}\right) \subset \bar{U}, \text { and } \sigma\left(\left.T\right|_{\mathcal{Y}}\right) \subset \bar{V} .
$$

It is well-known that

$$
\begin{aligned}
\text { Decomposable } & \Rightarrow \text { Bishop's property }(\beta) \\
& \Rightarrow \text { Dunford's property }(C) \Rightarrow \text { SVEP. }
\end{aligned}
$$

The converse implications do not hold, in general (see [20] for more details).

We say that Weyl's theorem holds for $T \in \mathcal{L}(\mathcal{H})$ if

$$
\sigma(T) \backslash \sigma_{w}(T)=\pi_{00}(T),
$$

where $\pi_{00}(T)=\{\lambda \in i \operatorname{so\sigma }(T): 0<\operatorname{dim} \operatorname{ker}(T-\lambda)<\infty\}$ and iso $\Delta$ denotes the set of all isolated points of $\Delta$. We say that Browder's theorem holds for $T \in \mathcal{L}(\mathcal{H})$ if $\sigma_{b}(T)=\sigma_{w}(T)$. We recall the definitions of some spectra;

$$
\sigma_{e a}(T):=\cap\left\{\sigma_{a}(T+K): K \in \mathcal{K}(\mathcal{H})\right\}
$$

is the essential approximate point spectrum, and

$$
\sigma_{a b}(T):=\cap\left\{\sigma_{a}(T+K): T K=K T \text { and } K \in \mathcal{K}(\mathcal{H})\right\}
$$


is the Browder essential approximate point spectrum. We put

$$
\pi_{00}(T):=\{\lambda \in \text { iso } \sigma(T): 0<\operatorname{dim} \operatorname{ker}(T-\lambda)<\infty\}
$$

and

$$
\pi_{00}^{a}(T):=\left\{\lambda \in \text { iso } \sigma_{a p}(T): 0<\operatorname{dim} \operatorname{ker}(T-\lambda)<\infty\right\} .
$$

For an operator $T \in \mathcal{L}(\mathcal{H})$, we say that

(i) a-Browder's theorem holds for $T$ if $\sigma_{e a}(T)=\sigma_{a b}(T)$;

(ii) a-Weyl's theorem holds for $T$ if $\sigma_{a p}(T) \backslash \sigma_{e a}(T)=\pi_{00}^{a}(T)$;

(iii) $T$ has the property $(w)$ if $\sigma_{a p}(T) \backslash \sigma_{e a}(T)=\pi_{00}(T)$.

It is known that

$$
\text { Property }(w) \Longrightarrow a \text {-Browder's theorem }
$$$$
\Downarrow
$$

Weyl's theorem $\Longleftarrow a$-Weyl's theorem.

We refer the reader to [1] for more details.

Let $T_{n}=\left.T\right|_{\operatorname{ran}\left(T^{n}\right)}$ for each nonnegative integer $n$; in particular, $T_{0}=T$. If $T_{n}$ is upper semi-Fredholm for some nonnegative integer $n$, then $T$ is called a upper semi$B$-Fredholm operator. In this case, by [7], $T_{m}$ is a upper semi-Fredholm operator and $\operatorname{ind}\left(T_{m}\right)=\operatorname{ind}\left(T_{n}\right)$ for each $m \geq n$. Thus, one can consider the index of $T$, denoted by $i n d_{B}(T)$, as the index of the semi-Fredholm operator $T_{n}$. Similarly, we define lower semi-B-Fredholm operators. We say that $T \in \mathcal{L}(\mathcal{H})$ is $B$-Fredholm if it is both upper and lower semi-B-Fredholm. In [7], Berkani proved that $T \in \mathcal{L}(\mathcal{H})$ is B-Fredholm if and only if $T=T_{1} \oplus T_{2}$ where $T_{1}$ is Fredholm and $T_{2}$ is nilpotent. Let $S B F_{+}^{-}(\mathcal{H})$ be the class of all upper semi- $B$-Fredholm operators such that $\operatorname{ind}_{B}(T) \leq 0$, and let

$$
\sigma_{S B F_{+}^{-}}(T):=\left\{\lambda \in \mathbb{C}: T-\lambda \notin S B F_{+}^{-}(\mathcal{H})\right\} .
$$

An operator $T \in \mathcal{L}(\mathcal{H})$ is called $B$-Weyl if it is B-Fredholm of index zero. The $B$-Weyl spectrum $\sigma_{B W}(T)$ of $T$ is defined by

$$
\sigma_{B W}(T):=\{\lambda \in \mathbb{C}: T-\lambda \text { is not a B-Weyl operator }\} .
$$

We say that $\lambda \in \sigma_{a p}(T)$ is a left pole of $T$ if it has finite ascent, i.e., $a(T)<\infty$ and $\operatorname{ran}\left(T^{a(T)+1}\right)$ is closed where $a(T)=\operatorname{dim} \operatorname{ker}(T)$. The notation $p_{0}(T)$ (respectively, $p_{0}^{a}(T)$ ) denotes the set of all poles (respectively, left poles) of $T$, while $\pi_{0}(T)$ (respectively, $\left.\pi_{0}^{a}(T)\right)$ is the set of all eigenvalues of $T$ which is an isolated point in $\sigma(T)$ (respectively, $\sigma_{a p}(T)$ ). 


\section{Main Results}

In this section, we study several properties of $m$-complex symmetric operators. Recall that an operator $N \in \mathcal{L}(\mathcal{H})$ is said to be nilpotent of order $n$ if $N^{n}=0$ and $N^{n-1} \neq 0$ for some positive integer $n$. It is well-known from [13, Theorem 5] that every nilpotent of order 2 is a complex symmetric (or 1-complex symmetric in our definition) operator. However if $T$ is nilpotent of order $n$ with $n>2$, then $T$ may not be a complex symmetric operator. We first give the following example of (strict) $m$-complex symmetric operators.

Example 3.1. Let $C$ be a conjugation given by $C\left(z_{1}, z_{2}, z_{3}\right)=\left(\overline{z_{3}}, \overline{z_{2}}, \overline{z_{1}}\right)$ on $\mathbb{C}^{3}$. If $N=\left(\begin{array}{lll}0 & 1 & 0 \\ 0 & 0 & 7 \\ 0 & 0 & 0\end{array}\right)$ on $\mathbb{C}^{3}$, then $N$ is nilpotent of order 3 and $N^{*} \neq C N C$. Since $N^{* 2}=C N^{2} C$, it follows that

$$
\begin{aligned}
\Delta_{3}(N)=\sum_{j=0}^{3}(-1)^{3-j}\left(\begin{array}{l}
3 \\
j
\end{array}\right) N^{* j} C N^{3-j} C & =-3 N^{* 2} C N C+3 N^{*} C N^{2} C \\
& =-3 C N^{3} C+3 N^{* 3}=0 .
\end{aligned}
$$

Hence $N$ is a strict 3-complex symmetric operator with conjugation $C$.

On the other hand, let $J$ be a conjugation given by $J\left(z_{1}, z_{2}, z_{3}\right)=\left(\overline{z_{1}}, \overline{z_{2}}, \overline{z_{3}}\right)$ on $\mathbb{C}^{3}$. Then $N$ is a 5 -complex symmetric operator with conjugation $J$ from [9]. Since $N^{3}=0$, we have

$$
\sum_{j=0}^{4}(-1)^{4-j}\left(\begin{array}{l}
4 \\
j
\end{array}\right) N^{* j} J N^{4-j} J=6 N^{* 2} J N^{2} J=\left(\begin{array}{ccc}
0 & 0 & 0 \\
0 & 0 & 0 \\
0 & 0 & 294
\end{array}\right) \neq 0 .
$$

Thus $N$ is not a 4-complex symmetric operator. Hence $N$ is a strict 5 -complex symmetric operator with conjugation $J$.

In the following theorem, we examine conditions for the operator $T+N$ to be a $(2 n+m-2)$-complex symmetric operator.

Theorem 3.2. Let $T \in \mathcal{L}(\mathcal{H})$ be strict $m$-complex symmetric with a conjugation $C$ and let $N$ be nilpotent of order $n>2$ with $T N=N T$. Then $T+N$ is a $(2 n+m-2)$-complex symmetric operator with conjugation $C$.

Proof. Let $R=T+N$ and $k=2 n+m-2$. Since

$$
\begin{aligned}
{[(a+b)-(c+d)]^{k} } & =[\{(a-c)+b\}-d)]^{k} \\
& =\sum_{i=0}^{k}(-1)^{i}\left(\begin{array}{c}
k \\
i
\end{array}\right)[(a-c)+b]^{k-i} d^{i} \\
& =\sum_{i=0}^{k} \sum_{j=0}^{k-i}(-1)^{i}\left(\begin{array}{c}
k \\
i
\end{array}\right)\left(\begin{array}{c}
k-i \\
j
\end{array}\right) b^{j}(a-c)^{k-i-j} d^{i} \\
& =\sum_{k_{1}+k_{2}+k_{3}=m}\left(\begin{array}{c}
k \\
k \\
k_{1}, k_{2}, k_{3}
\end{array}\right) b^{k_{3}}(a-c)^{k_{1}} d^{k_{2}}
\end{aligned}
$$


it follows that

$$
\begin{aligned}
\Delta_{k}(R) & =\sum_{k_{1}+k_{2}+k_{3}=k}\left(\begin{array}{c}
k \\
k_{1}, k_{2}, k_{3}
\end{array}\right) N^{* k_{3}} \Delta_{k_{1}}(T) C N^{k_{2}} C \\
& =\sum_{i=0}^{k} \sum_{j=0}^{k-i}(-1)^{i}\left(\begin{array}{c}
k \\
i
\end{array}\right)\left(\begin{array}{c}
k-i \\
j
\end{array}\right) N^{* j} \Delta_{k-i-j}(T) C N^{i} C .
\end{aligned}
$$

(i) If $j \geq n$ or $i \geq n$, then $N^{* j}=0$ and $N^{i}=0$. Hence (3.1) implies that $\Delta_{k}(R)=0$ due to the fact that $N^{n}=0$.

(ii) If $j<n$ and $i<n$, then

$$
\begin{aligned}
k-i-j & =(2 n+m-2)-i-j \\
& \geq 2 n+m-2-(n-1)-(n-1)=m .
\end{aligned}
$$

Thus $\Delta_{k-i-j}(T)=0$ and so $\Delta_{k}(R)=0$ from (3.1). Hence $T+N$ is a $(2 n+m-2)$ complex symmetric operator with conjugation $C$.

From Theorem 3.2, we also know that $T+N$ is not necessarily a strict $(2 n+m-2)$ complex symmetric operator. For example, if $T$ is a complex symmetric operator and $N$ is nilpotent of order $n>2$ with $T N=N T$, then $T=T+N+(-N)$ is not a strict $(4 n-3)$-complex symmetric operator.

Example 3.3. Let $N$ be a nilpotent operator of order $n>2$ with $N^{*} \neq C N C$. Then $I+N$ is an $(2 n-1)$-complex symmetric operator from Theorem 3.2. In particular, assume that $C$ is a conjugation given by $C\left(z_{1}, z_{2}, z_{3}\right)=\left(\overline{z_{1}}, \overline{z_{2}}, \overline{z_{3}}\right)$ on $\mathbb{C}^{3}$. If $R=$ $\left(\begin{array}{lll}1 & 1 & 0 \\ 0 & 1 & 3 \\ 0 & 0 & 1\end{array}\right)=I+N$ where $N=\left(\begin{array}{lll}0 & 1 & 0 \\ 0 & 0 & 3 \\ 0 & 0 & 0\end{array}\right)$ on $\mathbb{C}^{3}$, then $N^{3}=0$ and $N^{2} \neq 0$. Then we have $\Delta_{4}(R)=\Delta_{4}(N)=6 N^{* 2} C N^{2} C \neq 0$. Hence $R$ is a strict 5-complex symmetric operator from the previous note.

Remark 3.4. If we omit "strict" in Theorem 3.2, it is not necessarily that $T+N$ is a $(2 n+m-2)$-complex symmetric operator. For example, if $T=A \oplus 0$ and $N=0 \oplus Q$ where $A$ is an $m$-complex symmetric operator and $Q$ is a nilpotent operator of order $n$, then it is clear that $T$ is an $m$-complex symmetric operator, $N$ is a nilpotent operator of order $n$, and $T$ commutes with $N$. Hence $T+N=A \oplus Q$ is an $k$-complex symmetric operator for $k=\max \{m, 2 n-1\}$.

Recall that an operator $T \in \mathcal{L}(\mathcal{H})$ is said to be hyponormal if $T^{*} T \geq T T^{*}$. We next study some properties of $\Delta_{m}(T)$.

Proposition 3.5. Let $T$ be $(m+1)$-complex symmetric with a conjugation $C$. If $\Delta_{m}(T)$ is hyponormal, then $\operatorname{ker}\left(\Delta_{m}(T)-\lambda\right) \cap \operatorname{ker}\left(\Delta_{1}(T)-\lambda\right)=\{0\}$ for any nonzero $\lambda \in \mathbb{C}$.

Proof. If $x \in \operatorname{ker}\left(\Delta_{m}(T)-\lambda\right) \cap \operatorname{ker}\left(\Delta_{1}(T)-\lambda\right)$, then $\Delta_{m}(T) x=\Delta_{1}(T) x=\lambda x$. Since $\operatorname{ker}\left(\Delta_{m}(T)-\lambda\right) \subset \operatorname{ker}\left(\Delta_{m}(T)-\lambda\right)^{*}$, it follows from (1.2) that

$$
\begin{aligned}
0 & =\left\langle\Delta_{m+1}(T) x, x\right\rangle=\left\langle\left[T^{*} \Delta_{m}(T)-\Delta_{m}(T) C T C\right] x, x\right\rangle \\
& =\left\langle\Delta_{m}(T) x, T x\right\rangle-\left\langle C T C x, \Delta_{m}(T)^{*} x\right\rangle
\end{aligned}
$$




$$
\begin{aligned}
& =\lambda\left(\left\langle T^{*} x, x\right\rangle-\langle C T C x, x\rangle\right) \\
& =\lambda\left\langle\Delta_{1}(T) x, x\right\rangle=\lambda^{2}\|x\|^{2} .
\end{aligned}
$$

Hence we have $x=0$.

Corollary 3.6. Let $C$ be a conjugation operator on $\mathcal{H}$. Suppose that $H$ and $K$ are Hermitian operators which satisfy $H C K=K C H$ and $C S C \geq S$, where $S=i(H K-$ $K H)$. For an operator $T=H+i K$, if $T$ is 2 -complex symmetric with the conjugation $C$, then $\operatorname{ker}\left(\Delta_{1}(T)-\lambda\right)=\{0\}$ for any nonzero $\lambda \in \mathbb{C}$.

Proof. If $T=H+i K$, then

$$
\Delta_{1}(T)=T^{*}-C T C=(H-i K)-C(H+i K) C=\Delta_{1}(H)-i \Delta_{1}(K) .
$$

Since $\Delta_{1}(H)$ and $\Delta_{1}(K)$ are Hermitian, $H C K=K C H$, and $C S C \geq S$, it follows from (3.2) that

$$
\begin{aligned}
\Delta_{1}(T)^{*} \Delta_{1}(T)-\Delta_{1}(T) \Delta_{1}(T)^{*}= & 2 i\left[\Delta_{1}(K) \Delta_{1}(H)-\Delta_{1}(H) \Delta_{1}(K)\right] \\
= & 2 i[-(H K-K H)+(H C K-K C H) C \\
& +C(H C K-K C H)-C(H K-K H) C] \\
= & -2 i(H K-K H)+C[2 i(H K-K H) C] \\
= & 2(C S C-S) \geq 0 .
\end{aligned}
$$

Hence, $\Delta_{1}(T)$ is hyponormal and the proof follows by Proposition 3.5.

Lemma 3.7. Let $T$ be in $\mathcal{L}(\mathcal{H})$ and let $C$ be a conjugation on $\mathcal{H}$. If $T$ commutes with $N$ and $C N^{*} C$, then

$$
\Delta_{m}(T+N)=\sum_{j=0}^{m}\left(\begin{array}{c}
m \\
j
\end{array}\right) \Delta_{j}(T) \cdot \Delta_{m-j}(N)
$$

where $\Delta_{0}(T)=\Delta_{0}(N)=I$. In particular, if $T$ is complex symmetric with the conjugation $C$, then

for any $m \in \mathbb{N}$.

$$
\Delta_{m}(T+N)=\Delta_{m}(N)
$$

Proof. Let $R=T+N$. If $T$ commutes with $N$ and $C N^{*} C$, then it holds

$$
T \cdot C N^{* j} C=C N^{* j} C \cdot T \text { and } N \cdot C T^{* j} C=C T^{* j} C \cdot N
$$

for every positive integers $j$. Then (3.3) obviously holds for $m=1$. Suppose that (3.3) holds for $m$. Then (1.2) and (3.3) imply

$$
\begin{aligned}
\Delta_{m+1}(R)= & \left(T^{*}+N^{*}\right) \cdot \Delta_{m}(R)-\Delta_{m}(R) \cdot(C T C+C N C) \\
= & \sum_{j=0}^{m}\left(\begin{array}{c}
m \\
j
\end{array}\right)\left(T^{*}+N^{*}\right) \cdot \Delta_{j}(T) \cdot \Delta_{m-j}(N) \\
& -\sum_{j=0}^{m}\left(\begin{array}{c}
m \\
j
\end{array}\right) \Delta_{j}(T) \cdot \Delta_{m-j}(N) \cdot(C T C+C N C) \\
= & \sum_{j=0}^{m}\left(\begin{array}{c}
m \\
j
\end{array}\right)\left(T^{*} \cdot \Delta_{j}(T)-\Delta_{j}(T) \cdot C T C\right) \Delta_{m-j}(N)
\end{aligned}
$$




$$
\begin{aligned}
& +\sum_{j=0}^{m}\left(\begin{array}{c}
m \\
j
\end{array}\right) \Delta_{j}(T)\left(N^{*} \cdot \Delta_{m-j}(N)-\Delta_{m-j}(N) \cdot C N C\right) \\
= & \sum_{j=0}^{m}\left(\begin{array}{c}
m \\
j
\end{array}\right) \Delta_{j+1}(T) \cdot \Delta_{m-j}(N)+\sum_{j=0}^{m}\left(\begin{array}{c}
m \\
j
\end{array}\right) \Delta_{j}(T) \cdot \Delta_{m+1-j}(N) \\
= & \sum_{j=0}^{m+1}\left(\begin{array}{c}
m+1 \\
j
\end{array}\right) \Delta_{j}(T) \cdot \Delta_{m+1-j}(N) .
\end{aligned}
$$

Hence (3.3) holds for any positive integer $m$.

We will show the second statement. Suppose that $T$ is complex symmetric with the conjugation $C$. By induction, we prove that $\Delta_{m}(R)=\Delta_{m}(N)$ for any $m \in \mathbb{N}$. If $m=1$, it is obvious. Assume that $\Delta_{m-1}(R)=\Delta_{m-1}(N)$. Since $N$ and $C N^{*} C$ commute with $T$, it follows that

$$
\begin{aligned}
T^{*} \Delta_{m-1}(N) & =T^{*}\left[\sum_{j=0}^{m-1}(-1)^{m-1-j}\left(\begin{array}{c}
m-1 \\
j
\end{array}\right) N^{* j} C N^{m-1-j} C\right] \\
& =\left[\sum_{j=0}^{m-1}(-1)^{m-1-j}\left(\begin{array}{c}
m-1 \\
j
\end{array}\right) N^{* j} C N^{m-1-j} C\right] T^{*}=\Delta_{m-1}(N) T^{*} .
\end{aligned}
$$

Moreover, since $C T C=T^{*}$ and $T^{*}$ commutes with $\Delta_{m-1}(R)$, we obtain from (1.2) that

$$
\begin{aligned}
\Delta_{m}(R) & =R^{*} \Delta_{m-1}(R)-\Delta_{m-1}(R) C R C \\
& =\left(T^{*}+N^{*}\right) \Delta_{m-1}(N)-\Delta_{m-1}(N)(C T C+C N C) \\
& =\left(T^{*}+N^{*}\right) \Delta_{m-1}(N)-\Delta_{m-1}(N)\left(T^{*}+C N C\right) \\
& =N^{*} \Delta_{m-1}(N)-\Delta_{m-1}(N) C N C=\Delta_{m}(N) .
\end{aligned}
$$

So this completes the proof.

Proposition 3.8. Let $T \in \mathcal{L}(\mathcal{H})$ commute with $N$ and $C N^{*} C$ where $C$ is a conjugation on $\mathcal{H}$. If $T$ is $k$-complex symmetric for all $k$ with $0 \leq k \leq(2 l+k-2)$ and $N$ is a nilpotent of order $l$, then $T+N$ is $(2 l+k-2)$-complex symmetric. In particular, if $T$ is complex symmetric with the conjugation $C$, then $T+N$ is $(2 n-1)$-complex symmetric if and only if $N$ is a nilpotent of order $n$.

Proof. If $T$ is $m$-complex symmetric and $N$ is a nilpotent of order $n$, then $\Delta_{m}(T)=0$ and $\Delta_{2 n-1}(N)=0$ from [9]. Thus (3.3) and (1.2) implies $\Delta_{2 n+m-2}(T+N)=0$. Hence $T+N$ is $(2 n+m-2)$-complex symmetric. The remaining cases also hold by a similar method.

For the second statement, if $T$ is complex symmetric, then by (3.4), $T+N$ is $(2 n-1)$-complex symmetric if and only if $N$ is a nilpotent of order $n$.

We next consider the decomposability of $T+A$ and $T A$ where $T$ is $m$-complex symmetric operator and $A$ is an algebraic operator. For any set $G \subset \mathbb{C}$, we denote $G^{*}=\{\bar{z}: z \in G\}$. 
Theorem 3.9. Let $T \in \mathcal{L}(\mathcal{H})$ be an m-complex symmetric operator and $A$ be an algebraic operator of order $k$. If $R=T+A$ or $R=T A$ where $T$ commutes with $A$, then the following statements are equivalent:

(i) $T$ is decomposable.

(ii) $T^{*}$ has the property $(\beta)$.

(iii) $R$ is decomposable.

(iv) $R^{*}$ has the property $(\beta)$.

Proof. Since the proof of (i) $\Leftrightarrow$ (ii) and (iii) $\Leftrightarrow$ (iv) follow from [9, Theorem 4.7], we only consider the following implication (ii) $\Leftrightarrow$ (iv).

(1) In the case $R=T+A$. Assume that $T^{*}$ has the property $(\beta)$. Since $A$ is an algebraic operator of order $k$, there exists a nonconstant polynomial $p(\lambda)=$ $\left(\lambda-\gamma_{1}\right)\left(\lambda-\gamma_{2}\right)\left(\lambda-\gamma_{3}\right) \cdots\left(\lambda-\gamma_{k}\right)$ such that $p(A)=0$. Set $p_{0}(\lambda)=1$ and $p_{j}(\lambda)=$ $\left(\lambda-\gamma_{1}\right)\left(\lambda-\gamma_{2}\right) \cdots\left(\lambda-\gamma_{j}\right)$ for $j=1,2, \cdots, k$. Let $G$ be an open set in $\mathbb{C}$ and $f_{n}: G \rightarrow \mathcal{H}$ be a sequence of analytic functions such that

$$
\lim _{n \rightarrow \infty}\left\|\left(T^{*}+A^{*}-z\right) f_{n}(z)\right\|_{K}=0
$$

for every compact set $K$ in $D$. Fix any compact subset $K$ of $D$. Since

$$
\left(A^{*}-\overline{\gamma_{1}}\right)\left(A^{*}-\overline{\gamma_{2}}\right)\left(A^{*}-\overline{\gamma_{3}}\right) \cdots\left(A^{*}-\overline{\gamma_{k}}\right)=0
$$

$p_{k-1}(A)^{*} A^{*}=\overline{\gamma_{k}} p_{k-1}(A)^{*}$. This gives that

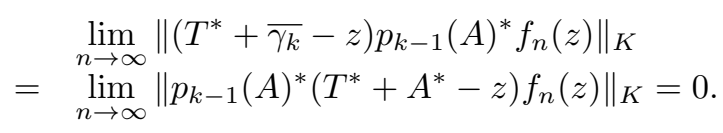

Moreover, since $T^{*}+\overline{\gamma_{k}}$ has the property $(\beta)$, we have

$$
\lim _{n \rightarrow \infty}\left\|p_{k-1}(A)^{*} f_{n}(z)\right\|_{K}=0 .
$$

Equations (3.5) and (3.7) imply that

$$
=\quad \begin{aligned}
& \lim _{n \rightarrow \infty}\left\|\left(T^{*}+\overline{\gamma_{k-1}}-z\right) p_{k-2}(A)^{*} f_{n}(z)\right\|_{K} \\
& \lim _{n \rightarrow \infty}\left\|p_{k-2}(A)^{*}\left(T^{*}+A^{*}-z\right) f_{n}(z)\right\|_{K}=0 .
\end{aligned}
$$

Since $T^{*}+\overline{\gamma_{k-1}}$ has the property $(\beta)$, we get that $\lim _{n \rightarrow \infty}\left\|p_{k-2}(A)^{*} f_{n}(z)\right\|_{K}=$ 0 . Hence, by induction we get that $\lim _{n \rightarrow \infty}\left\|f_{n}(z)\right\|_{K}=0$. Therefore, $R^{*}$ has the property $(\beta)$.

(2) In the case $R=T A$. Assume that $T^{*}$ has the property $(\beta)$. Let $G$ be an open set in $\mathbb{C}$ and $f_{n}: G \rightarrow \mathcal{H}$ be a sequence of analytic functions such that

$$
\lim _{n \rightarrow \infty}\left\|\left(R^{*}-z\right) f_{n}(z)\right\|_{K}=\lim _{n \rightarrow \infty}\left\|\left(T^{*} A^{*}-z\right) f_{n}(z)\right\|_{K}=0
$$

for every compact set $K$ in $D$. Thus, it holds that

$$
\lim _{n \rightarrow \infty}\left\|\left(A^{*}-\overline{\gamma_{k}}\right) T^{*} f_{n}(z)+\overline{\gamma_{k}} T^{*} f_{n}(z)-z f_{n}(z)\right\|_{K}=0 .
$$

Since $T^{*} A^{*}=A^{*} T^{*}$ and $p(A)^{*}=0$, we obtain from (3.9) that

$$
\lim _{n \rightarrow \infty}\left\|\left(\overline{\gamma_{k}} T^{*}-z\right) p_{k-1}(A)^{*} f_{n}(z)\right\|_{K}=0
$$


In addition, since $\overline{\gamma_{k}} T^{*}$ has the property $(\beta),(3.10)$ implies that

$$
\lim _{n \rightarrow \infty}\left\|p_{k-1}(A)^{*} f_{n}(z)\right\|_{K}=0 .
$$

Then we get from (3.8) that

$$
\lim _{n \rightarrow \infty}\left\|\left(A^{*}-\overline{\gamma_{k-1}}\right) T^{*} f_{n}(z)+\overline{\gamma_{k-1}} T^{*} f_{n}(z)-z f_{n}(z)\right\|_{K}=0 .
$$

Since $T^{*} A^{*}=A^{*} T^{*}$ and $p(A)^{*}=0$, we obtain from (3.12) that

$$
\lim _{n \rightarrow \infty}\left\|\left(\overline{\gamma_{k-1}} T^{*}-z\right) p_{k-2}(A)^{*} f_{n}(z)\right\|_{K}=0 .
$$

Moreover, since $\overline{\gamma_{k-1}} T^{*}$ has the property $(\beta),(3.13)$ implies that

$$
\lim _{n \rightarrow \infty}\left\|p_{k-2}(A)^{*} f_{n}(z)\right\|_{K}=0 .
$$

Hence, by induction we get $\lim _{n \rightarrow \infty}\left\|f_{n}(z)\right\|_{K}=0$, and so $R^{*}$ has the property $(\beta)$. The converse implication holds by similar arguments above. So this completes the proof.

We observe that the order $k$ of $A$ played a role to eliminate $A$ in the proof of Theorem 3.9. Moreover, we need an $m$-complex symmetric operator to prove (i) $\Leftrightarrow$ (ii)(see [9, Theorem 4.7]).

Corollary 3.10. Let $T \in \mathcal{L}(\mathcal{H})$ be a complex symmetric operator and $A$ be an algebraic operator of order $k$. If $R=T+A$ or $R=T A$ where $T$ commutes with $A$, then the following statements are equivalent:

(i) $T$ is decomposable.

(ii) $T^{*}$ has the property $(\beta)$.

(iii) $T$ has the property $(\beta)$.

(iv) $R$ is decomposable.

(v) $R^{*}$ has the property $(\beta)$.

(vi) $R$ has the property $(\beta)$.

Proof. Suppose that $T$ is a complex symmetric operator. Since the implications (i) $\Rightarrow$ (ii) and (i) $\Rightarrow$ (iii) hold by [20, Theorems 1.2.29 and 2.2.5], we consider the reverse implications. If $T^{*}$ has the property $(\beta)$, then $T$ is decomposable from [9]. If $T$ has the property $(\beta)$, then $T$ is decomposable from [19]. Therefore, we have (i) $\Leftrightarrow($ ii) $\Leftrightarrow($ iii). Moreover, we get that (iii) $\Leftrightarrow(\mathrm{vi})$ by a similar method. Hence we get this result from Theorem 3.9 .

Recall that an operator $T \in \mathcal{L}(\mathcal{H})$ is called a 2-normal operator if $T$ is unitarily equivalent to an operator matrix of the form $\left(\begin{array}{ll}T_{1} & T_{2} \\ T_{3} & T_{4}\end{array}\right) \in \mathcal{L}(\mathcal{H} \oplus \mathcal{H})$ where $T_{i}$ are mutually commuting normal operators.

Example 3.11. Let $R \in \mathcal{L}(\mathcal{H} \oplus \mathcal{H})$ be a 2-normal operator. Then $R$ is complex symmetric from [16] and $R$ is unitarily equivalent to $\left(\begin{array}{cc}N_{1} & N_{2} \\ 0 & N_{3}\end{array}\right)$. If $N_{1} N_{2}=N_{2} N_{3}$, then $\left(\begin{array}{cc}N_{1} & 0 \\ 0 & N_{3}\end{array}\right)$ and $\left(\begin{array}{cc}0 & N_{2} \\ 0 & 0\end{array}\right)$ commute and $\left(\begin{array}{cc}0 & N_{2} \\ 0 & 0\end{array}\right)$ is nilpotent of order 2. Moreover, 
since $N_{1}{ }^{*} \oplus N_{3}{ }^{*}$ has the property $(\beta)$, it follows that $\left(\begin{array}{cc}N_{1} & N_{2} \\ 0 & N_{3}\end{array}\right)$ is decomposable from Theorem 3.9. Hence $R$ is decomposable.

Let us recall that for an operator $T \in \mathcal{L}(\mathcal{H})$, a closed subspace $\mathcal{M} \subset \mathcal{H}$ is invariant for $T$ if $T \mathcal{M} \subset \mathcal{M}$, and it is hyperinvariant for $T$ if it is invariant for every operator in the commutant $\{T\}^{\prime}=\{S \in \mathcal{L}(\mathcal{H}): T S=S T\}$ of $T$. A subspace $\mathcal{M}$ of $\mathcal{H}$ is nontrivial if it is different from $\{0\}$ and $\mathcal{H}$. As some applications of Theorem 3.9, we get the following corollary.

Corollary 3.12. Let $R=T+A$ or $R=T A$ be in $\mathcal{L}(\mathcal{H})$ where $T$ is an $m$-complex symmetric operator and $A$ is an algebriac operator of order $k$ with $T A=A T$. If $T^{*}$ has the property $(\beta)$, then the following statements hold:

(i) $R$ and $R^{*}$ have the property $(\beta)$ and the single-valued extension property.

(ii) If $\sigma(R)$ has nonempty interior, then $R$ has a nontrivial invariant subspace.

(iii) $H_{R}(F)$ is a hyperinvariant subspace for $R$.

(iv) If $f$ is any function analytic on a neighborhood of $\sigma(R)$, then both Weyl's and Browder's theorems hold for $f(R)$ and

$$
\sigma_{w}(f(R))=\sigma_{b}(f(R))=f\left(\sigma_{w}(R)\right)=f\left(\sigma_{b}(R)\right) .
$$

Proof. (i) From [20], we know that $R$ is decomposable if and only if $R$ and $R^{*}$ have the property $(\beta)$. Hence this completes the proof.

(ii) Since $T^{*}$ has the property $(\beta)$, it follows from Theorem 3.9 that $R$ is decomposable. Moreover, since $R$ has the property $(\beta)$ by [20] and $\sigma(R)$ has nonempty interior, the proof follows from [12, Theorem 2.1].

(iii) If $T^{*}$ has the property $(\beta)$, then $R$ is decomposable from Theorem 3.9. Therefore $H_{R}(F)$ is a spectral maximal space of $R$ by [11, Proposition 3.8] and [20, Theorem 1.2.29]. Hence $H_{R}(F)$ is a hyperinvariant subspace for $R$.

(iv) Since $f(R)$ is decomposable from [20, p 145], it follows that $f(R)$ is clearly subscalar. Hence $f(R)$ satisfies Weyl's theorem from [1, p 175]. Moreover, since $f(R)$ has the single-valued extension property, Browder's theorem holds for $f(R)$ and the last relations are satisfied from [1, Theorem 3.71].

Proposition 3.13. Let $R=T+N$ where $T \in \mathcal{L}(\mathcal{H})$ is an m-complex symmetric operator with a conjugation $C$ and $N$ is a nilpotent operator of order $n$ with $T N=N T$. Then the following arguments hold;

(i) If $T^{*}$ has the single-valued extension property, then $R$ and $R^{*}$ has the single-valued extension property.

(ii) If $T$ has Dunford's property $(C)$ and $\sigma_{T}(x) \subset \sigma_{R}\left(N^{n-1} x\right) \cap \sigma_{R}(x)$ for all $x \in \mathcal{H}$, then $R$ has Dunford's property $(C)$.

Proof. (i) Let $R=T+N$. If $T$ is $m$-complex symmetric and $T^{*}$ has the single-valued extension property, then $T$ has the single-valued extension property from $[9$, Theorem 4.10]. Let $G$ be an open set in $\mathbb{C}$ and let $f: G \rightarrow \mathcal{H}$ be an analytic function such that $(R-z) f(z) \equiv 0$ on $G$, which implies

$$
(T-z) f(z)+N f(z)=0 .
$$


Since $N^{n}=0$ and $T N=N T$, it follows that $(T-z) N^{n-1} f(z)=0$. Since $T$ has the single-valued extension property, we have $N^{n-1} f(z)=0$. Moreover, (3.15) implies $(T-z) N^{n-2} f(z)=0$. Since $T$ has the single-valued extension property, we get that $N^{n-2} f(z)=0$. By similar process, we obtain that $f(z)=0$. Hence $R$ has the singlevalued extension property. Similarly, we get that $R^{*}$ have the single-valued extension property. Hence $R$ and $R^{*}$ have the single-valued extension property.

(ii) Let $T$ have Dunford's property $(C)$ and $\sigma_{T}(x) \subset \sigma_{R}\left(N^{n-1} x\right)$ for all $x \in \mathcal{H}$. Then it suffices to show that $\sigma_{R}\left(N^{n-1} x\right) \subset \sigma_{T}(x)$. Indeed, we assume $z_{0} \in \rho_{T}(x)$. Then there is an $\mathcal{H}$-valued analytic function $f(z)$ in a neighborhood $D$ of $z_{0}$ such that $(T-z) f(z)=x$ for every $z \in D$. Since $T N=N T$ and $N^{n}=0$, it follows that

$$
(R-z) N^{n-1} f(z)=(T-z) N^{n-1} f(z) \equiv N^{n-1} x \text { on } D .
$$

Since $N^{n-1} f(z)$ is analytic on $D$, we get $z_{0} \in \rho_{R}\left(N^{n-1} x\right)$. Hence $\sigma_{R}\left(N^{n-1} x\right) \subset$ $\sigma_{T}(x)$. Thus $\sigma_{T}(x)=\sigma_{R}\left(N^{n-1} x\right)$. Therefore, we have $N^{n-1} H_{R}(F)=H_{T}(F)$. Since $N^{n-1} H_{R}(F) \subset H_{R}(F)$, it follows that $H_{T}(F) \subset H_{R}(F)$ where $F$ is a closed subset of C. Moreover, since $\sigma_{T}(x) \subset \sigma_{R}(x)$ for all $x \in \mathcal{H}$, it follows that $H_{R}(F) \subset H_{T}(F)$ and so $H_{R}(F)=H_{T}(F)$ is closed for each closed subset $F$ of $\mathbb{C}$. Hence $R$ has Dunford's property $(C)$. This completes the proof.

For an operator $T \in \mathcal{L}(\mathcal{H})$, the quasinilpotent part of $T$ is defined by

$$
H_{0}(T):=\left\{x \in \mathcal{H}: \lim _{n \rightarrow \infty}\left\|T^{n} x\right\|^{\frac{1}{n}}=0\right\} .
$$

Then $H_{0}(T)$ is a linear (not necessarily closed) subspace of $\mathcal{H}$. We remark from [3] that if $T$ has the single-valued extension property, then

$$
H_{0}(T-\lambda)=\left\{x \in \mathcal{H}: \lim _{n \rightarrow \infty}\left\|(T-\lambda)^{n} x\right\|^{\frac{1}{n}}=0\right\}=H_{T}(\{\lambda\})
$$

for all $\lambda \in \mathbb{C}$. It is well known from [1] and [3] that if $H_{0}(T-\lambda)=\{0\}$ for all $\lambda \in \mathbb{C}$, then $T$ has the single-valued extension property.

Corollary 3.14. Let $R=T+N$ be in $\mathcal{L}(\mathcal{H})$ with the same hypotheses as in Proposition 3.13. If $T^{*}$ has the single-valued extension property, then the following properties hold:

(i) $\sigma(R)=\sigma_{s u}(R)=\sigma_{a p}(R)=\sigma_{s e}(R)$.

(ii) $\sigma_{e s}(R)=\sigma_{b}(R)=\sigma_{w}(R)=\sigma_{e}(R)$.

(iii) $H_{0}(R-\lambda)=H_{R}(\{\lambda\})$ and $H_{R^{*}}(\{\lambda\})=H_{0}\left(R^{*}-\lambda\right)$ for all $\lambda \in \mathbb{C}$.

Proof. Since $T^{*}$ has the single-valued extension property, it follows that $R$ and $R^{*}$ have the single-valued extension property from Proposition 3.13. Hence the proof follows from [1, Corollaries 2.45 and 3.53], and [3, Theorem 1.5].

We next state various spectral relations of $m$-complex symmetric operators.

Lemma 3.15. If $T$ is an $m$-complex symmetric operator, then the following relations hold;

(i) $\sigma_{p}(T) \subseteq \sigma_{p}\left(T^{*}\right)^{*}, \sigma_{a p}(T) \subset \sigma_{a p}\left(T^{*}\right)^{*}, \Gamma\left(T^{*}\right)^{*} \subseteq \Gamma(T), \sigma_{s u}\left(T^{*}\right)^{*} \subseteq \sigma_{s u}(T)$, and

$$
\sigma(T)=\sigma_{a p}\left(T^{*}\right)^{*}=\sigma_{s u}(T) .
$$


(ii) $\sigma_{l e}(T) \subseteq \sigma_{l e}\left(T^{*}\right)^{*}, \sigma_{r e}\left(T^{*}\right)^{*} \subseteq \sigma_{r e}(T)$, and $\sigma_{e}(T)=\sigma_{r e}(T)$.

(iii) If $T^{*}$ has the single-valued extension property, then

$$
\sigma(T)=\sigma_{a p}(T)=\sigma_{a p}\left(T^{*}\right)^{*}=\sigma\left(T^{*}\right)^{*} .
$$

Proof. (i) From [9, Theorem 4.1], $\sigma_{p}(T) \subseteq \sigma_{p}\left(T^{*}\right)^{*}$ and $\sigma_{a p}(T) \subset \sigma_{a p}\left(T^{*}\right)^{*}$. Since $\Gamma(S)^{*}=\sigma_{p}\left(S^{*}\right)$ and $\sigma_{s u}(S)^{*}=\sigma_{a p}\left(S^{*}\right)$ for any $S \in \mathcal{L}(\mathcal{H}), \Gamma\left(T^{*}\right)^{*} \subseteq \Gamma(T)$ and $\sigma_{s u}\left(T^{*}\right)^{*} \subseteq \sigma_{s u}(T)$. On the other hand, since $T$ is an $m$-complex symmetric operator, it follows from [17, Corollary, page 222] that $\sigma(T)=\sigma_{a p}(T) \cup \sigma_{a p}\left(T^{*}\right)^{*} \subseteq \sigma_{a p}\left(T^{*}\right)^{*}$. Since $\sigma_{s u}(S)^{*}=\sigma_{a p}\left(S^{*}\right)$ for any $S \in \mathcal{L}(\mathcal{H})$, we get that $\sigma(T) \subseteq \sigma_{a p}\left(T^{*}\right)^{*}=\sigma_{s u}(T) \subset$ $\sigma(T)$. Hence we obtain

$$
\sigma(T)=\sigma_{a p}\left(T^{*}\right)^{*}=\sigma_{s u}(T) .
$$

(ii) If $\lambda \in \sigma_{l e}(T)$, then there exists a sequence $\left\{x_{n}\right\}$ of unit vectors in $\mathcal{H}$ such that $\left\{x_{n}\right\}$ weakly converges to 0 and $\lim _{n \rightarrow \infty}\left\|(T-\lambda) x_{n}\right\|=0$ for any $T \in \mathcal{L}(\mathcal{H})$. Then we have $\lim _{n \rightarrow \infty}(C T C-\bar{\lambda}) C x_{n}=0$. Since $T$ is an $m$-complex symmetric operator with conjugation $C$, it follows that

$$
\begin{aligned}
0 & =\lim _{n \rightarrow \infty}\left\|\left(\sum_{j=0}^{m}(-1)^{m-j}\left(\begin{array}{c}
m \\
j
\end{array}\right) T^{* j} C T^{m-j} C\right) C x_{n}\right\| \\
& =\lim _{n \rightarrow \infty}\left\|\left(\sum_{j=0}^{m}(-1)^{m-j}\left(\begin{array}{c}
m \\
j
\end{array}\right) T^{* j} \bar{\lambda}^{m-j}\right) C x_{n}\right\| \\
& =\lim _{n \rightarrow \infty}\left\|\left(T^{*}-\bar{\lambda}\right)^{m} C x_{n}\right\| .
\end{aligned}
$$

Moreover, since $\left\{x_{n}\right\}$ weakly converges to $0,\left\{C x_{n}\right\}$ weakly converges to 0 . Hence we get that $\sigma_{l e}(T) \subseteq \sigma_{l e}\left(T^{*}\right)^{*}$. Since $\sigma_{r e}(S)^{*}=\sigma_{l e}\left(S^{*}\right)$ for any $S \in \mathcal{L}(\mathcal{H})$, it follows that $\sigma_{r e}\left(T^{*}\right)^{*} \subseteq \sigma_{r e}(T)$. Moreover, since $\sigma_{e}(S)=\sigma_{l e}(S) \cup \sigma_{r e}(S)$ for any $S \in \mathcal{L}(\mathcal{H})$, we obtain that

$$
\sigma_{e}(T)=\sigma_{l e}(T) \cup \sigma_{r e}(T) \subseteq \sigma_{l e}\left(T^{*}\right)^{*} \cup \sigma_{r e}(T)=\sigma_{r e}(T) .
$$

Since $\sigma_{r e}(S) \subseteq \sigma_{e}(S)$ for any $S \in \mathcal{L}(\mathcal{H})$, we obtain that $\sigma_{r e}(T)=\sigma_{e}(T)$.

(iii) If $T^{*}$ has the single-valued extension property, then $T$ has the single-valued extension property from [9]. Note that $\sigma(S)^{*}=\sigma\left(S^{*}\right)$ and $\sigma_{s u}(S)^{*}=\sigma_{a p}\left(S^{*}\right)$ for any $S \in \mathcal{L}(\mathcal{H})$. Since $T$ and $T^{*}$ have the single-valued extension property, it follows from [20] that $\sigma(T)^{*}=\sigma\left(T^{*}\right)=\sigma_{s u}\left(T^{*}\right)=\sigma_{a p}(T)^{*}$. Moreover, since $\sigma_{a p}(T) \subset \sigma_{a p}\left(T^{*}\right)^{*}$ by (i), it follows that $\sigma(T)=\sigma_{a p}(T) \subseteq \sigma_{a p}\left(T^{*}\right)^{*} \subseteq \sigma\left(T^{*}\right)^{*}=\sigma(T)$. Hence we get

$$
\sigma(T)=\sigma_{a p}(T)=\sigma_{a p}\left(T^{*}\right)^{*}=\sigma\left(T^{*}\right)^{*} .
$$

This completes the proof.

Proposition 3.16. Let $T \in \mathcal{L}(\mathcal{H})$ be an m-complex symmetric operator and $N$ be a nilpotent operator of order $n$ with $T N=N T$. If $R=T+N$, then the following properties hold:

(i) $\sigma_{p}(R) \subset \sigma_{p}\left(T^{*}\right)^{*} \cup\{0\}, \Gamma\left(R^{*}\right)^{*} \subset \Gamma(T) \cup\{0\}, \sigma_{a p}(R) \subseteq \sigma_{a p}\left(T^{*}\right)^{*} \cup\{0\}$, and $\sigma_{a p}(R) \subseteq \sigma(T) \cup\{0\}$.

(ii) $\sigma_{l e}(R) \subset \sigma_{l e}(T)$ and $\sigma_{r e}\left(R^{*}\right)^{*} \subset \sigma_{r e}\left(T^{*}\right)^{*}$. In addition, if $T^{*}$ is an $m$-complex symmetric operator, then $\sigma_{e}(R) \subseteq \sigma_{e}(T)$. 
Proof. (i) Assume that $R=T+N$ where $T$ is an $m$-complex symmetric operator, $N^{n}=0$, and $T N=N T$. Since $T$ commutes with $N$, it follows from Lemma 3.15 and [20, Page 256] that

$$
\sigma_{a p}(R) \subseteq \sigma_{a p}(T)+\sigma_{a p}(N) \subseteq \sigma_{a p}\left(T^{*}\right)^{*} \cup\{0\} .
$$

Hence $\sigma_{a p}(R) \subseteq \sigma(T) \cup\{0\}$ from Lemma 3.15. By the similar method, we get that $\sigma_{p}(R) \subset \sigma_{p}\left(T^{*}\right)^{*} \cup\{0\}$. On the other hand, since $\Gamma(S)^{*}=\sigma_{p}\left(S^{*}\right)$ for any $S \in \mathcal{L}(\mathcal{H})$ and the previous result, we conclude that $\Gamma\left(R^{*}\right)^{*} \subset \Gamma(T) \cup\{0\}$.

(ii) If $\lambda \in \sigma_{l e}(R)$, then there exists a sequence $\left\{x_{i}\right\}$ of unit vectors in $\mathcal{H}$ such that $\left\{x_{i}\right\}$ weakly converges to 0 and $\lim _{i \rightarrow \infty}\left\|(R-\lambda) x_{i}\right\|=0$. Put $y_{i}=\frac{N^{n-1} x_{i}}{\left\|N^{n-1} x_{i}\right\|}$ for some $n \geq 1$. Since $T$ commutes with $N$ and $N^{n}=0$, it follows that

$$
\begin{aligned}
\lim _{i \rightarrow \infty}\left\|(T-\lambda) y_{i}\right\| & =\lim _{i \rightarrow \infty}\left\|(T-\lambda) \frac{N^{n-1} x_{i}}{\left\|N^{n-1} x_{i}\right\|}\right\| \\
& =\lim _{i \rightarrow \infty}\left\|N^{n-1}(T+N-\lambda) \frac{x_{i}}{\left\|N^{n-1} x_{i}\right\|}\right\| \\
& =\lim _{i \rightarrow \infty}\left\|N^{n-1}(R-\lambda) \frac{x_{i}}{\left\|N^{n-1} x_{i}\right\|}\right\|=0 .
\end{aligned}
$$

In addition, if $\left\{x_{i}\right\}$ weakly converges to 0 , then $\left\{y_{i}\right\}$ weakly converges to 0 . Therefore $\lambda \in \sigma_{l e}(T)$. So, $\sigma_{l e}(R) \subseteq \sigma_{l e}(T)$. Since $\sigma_{r e}(S)^{*}=\sigma_{l e}\left(S^{*}\right)$ for any $S \in \mathcal{L}(\mathcal{H})$, we obtain $\sigma_{r e}\left(R^{*}\right)^{*} \subset \sigma_{r e}\left(T^{*}\right)^{*}$. If $T^{*}$ is an $m$-complex symmetric operator, then we get $\sigma_{l e}\left(R^{*}\right) \subset \sigma_{l e}\left(T^{*}\right)$ in a similar way. Thus $\sigma_{e}(R)=\sigma_{l e}(R) \cup \sigma_{r e}(R) \subseteq \sigma_{l e}(T) \cup \sigma_{r e}(T)=$ $\sigma_{e}(T)$. Hence $\sigma_{e}(R) \subseteq \sigma_{e}(T)$. This completes the proof.

Finally, we deal with Weyl type theorems for $m$-complex symmetric operators.

Theorem 3.17. Let $T \in \mathcal{L}(\mathcal{H})$ be $m$-complex symmetric. Suppose that $T^{*}$ has the single-valued extension property. Then the following statements are equivalent:

(i) $T^{*}$ satisfies a-Weyl's theorem.

(ii) $T^{*}$ satisfies Weyl's theorem.

(iii) $T^{*}$ has the property $(w)$.

In addition, the following statements are equivalent.

(iv) $T^{*}$ satisfies generalized a-Weyl's theorem.

(v) $T^{*}$ satisfies generalized Weyl's theorem.

Proof. (a) Suppose that $T^{*}$ satisfies Weyl's theorem. Since $T$ is $m$-complex symmetric, it follows from Lemma 3.15 that $\sigma_{a p}\left(T^{*}\right)^{*}=\sigma(T)=\sigma\left(T^{*}\right)^{*}$ and so $\sigma_{a p}\left(T^{*}\right)=\sigma\left(T^{*}\right)$. On the other hand, since $\sigma_{e a}\left(T^{*}\right) \subset \sigma_{w}\left(T^{*}\right)$ is obvious, it suffices to show $\sigma_{w}\left(T^{*}\right) \subset$ $\sigma_{e a}\left(T^{*}\right)$. Indeed, if $\lambda \notin \sigma_{e a}\left(T^{*}\right)$, then $T^{*}-\lambda$ is semi-Fredholm and $\operatorname{ind}\left(T^{*}-\lambda\right) \leq 0$. Since $T^{*}$ has the single-valued extension property, it follows from [9] and [1] that $T=\left(T^{*}\right)^{*}$ has the single-valued extension property and $\operatorname{ind}\left(T^{*}-\lambda\right) \geq 0$ for every $\lambda \notin \sigma_{e a}\left(T^{*}\right)$, respectively. Therefore $\operatorname{ind}\left(T^{*}-\lambda\right)=0$ for every $\lambda \notin \sigma_{e a}\left(T^{*}\right)$. Thus $\lambda \notin \sigma_{w}\left(T^{*}\right)$. Hence $\sigma_{e a}\left(T^{*}\right)=\sigma_{w}\left(T^{*}\right)$. This gives that

$$
\pi_{00}^{a}\left(T^{*}\right)=\pi_{00}\left(T^{*}\right)=\sigma\left(T^{*}\right) \backslash \sigma_{w}\left(T^{*}\right)=\sigma_{a p}\left(T^{*}\right) \backslash \sigma_{e a}\left(T^{*}\right) .
$$

Hence $a$-Weyl's theorem holds for $T^{*}$. Similarly, since $\pi_{00}^{a}\left(T^{*}\right)=\pi_{00}\left(T^{*}\right)$, we can show that (i) $\Leftrightarrow$ (iii). It is clear that (i) $\Rightarrow$ (ii). So we have this result. 
(b) By [5, Theorem 3.7], it suffices to prove that (ii) $\Rightarrow$ (i). Suppose that $T^{*}$ satisfies generalized Weyl's theorem. Then $\sigma_{B W}\left(T^{*}\right)=\sigma\left(T^{*}\right) \backslash \pi_{0}\left(T^{*}\right)$. Since $T$ is $m$-complex symmetric, it follows from Lemma 3.15 that $\sigma_{a p}\left(T^{*}\right)=\sigma\left(T^{*}\right)$ and so

$$
\sigma_{B W}\left(T^{*}\right)=\sigma\left(T^{*}\right) \backslash \pi_{0}\left(T^{*}\right)=\sigma_{a p}\left(T^{*}\right) \backslash \pi_{0}^{a}\left(T^{*}\right) .
$$

Hence it suffices to show that $\sigma_{S B F_{+}^{-}}\left(T^{*}\right)=\sigma_{B W}\left(T^{*}\right)$. If $\lambda \notin \sigma_{S B F_{+}^{-}}\left(T^{*}\right)$, then $T^{*}-\lambda$ is semi-B-Fredholm and $\operatorname{ind}_{B}\left(T^{*}-\lambda\right) \leq 0$. Since $T$ is $m$-complex symmetric operator and $T^{*}$ has the single-valued extension property, it follows from [1] that $\operatorname{ind}_{B}\left(T^{*}-\lambda\right) \geq 0$ for every $\lambda \notin \sigma_{S B F_{+}^{-}}\left(T^{*}\right)$. Thus $i n d_{B}\left(T^{*}-\lambda\right)=0$ for every $\lambda \notin \sigma_{S B F_{+}^{-}}\left(T^{*}\right)$. Therefore $\sigma_{S B F_{+}^{-}}\left(T^{*}\right) \supset \sigma_{B W}\left(T^{*}\right)$. Since $\sigma_{S B F_{+}^{-}}\left(T^{*}\right) \subset \sigma_{B W}\left(T^{*}\right)$ is clear, we obtain that

$$
\sigma_{S B F_{+}^{-}}\left(T^{*}\right)=\sigma_{B W}\left(T^{*}\right)=\sigma_{a p}\left(T^{*}\right) \backslash \pi_{00}^{a}\left(T^{*}\right) .
$$

Hence the generalized $a$-Weyl's theorem holds for $T^{*}$.

Corollary 3.18. Let $T \in \mathcal{L}(\mathcal{H})$ be an $m$-complex symmetric operator. Then the following arguments are equivalent:

(i) $T^{*}$ satisfies Browder's theorem.

(ii) $T^{*}$ satisfies a-Browder's theorem.

(iii) $T^{*}$ satisfies the generalized Browder's theorem.

(iv) $T^{*}$ satisfies the generalized a-Browder's theorem.

Proof. Since it is well known that (i) $\Leftrightarrow$ (iii) and (ii) $\Leftrightarrow$ (iv) from [4, Theorem 2.1 and Theorem 2.2], we only consider (iii) $\Leftrightarrow$ (iv). Since $\sigma\left(T^{*}\right)=\sigma_{a p}\left(T^{*}\right)$ from Lemma 3.15 , we have $p_{0}\left(T^{*}\right)=p_{0}^{a}\left(T^{*}\right)$. Moreover, $\sigma_{S B F_{+}^{-}}\left(T^{*}\right)=\sigma_{B W}\left(T^{*}\right)$ as in the proof of Theorem 3.17. Using these results, we get that (iii) $\Leftrightarrow$ (iv). This completes the proof.

\section{References}

[1] Aiena, P., Fredholm and local spectral theory with applications to multipliers, Kluwer Academic Publ., 2004.

[2] Agler, J., Sub-Jordan operators: Bishop's theorem, spectral inclusion, and spectral sets, J. Oper. Theory, 7(1982), no. 2, 373-395.

[3] Aiena, P., Colasante, M.L., González, M., Operators which have a closed quasi-nilpotent part, Proc. Amer. Math. Soc., 130(2002), 2701-2710.

[4] Amouch, M., Zguitti, H., On the equivalence of Browder's and generalized Browder's theorem, Glasgow Math. J., 48(2006), 179-185.

[5] Berkani, M., Koliha, J.J., Weyl type theorems for bounded linear operators, Acta Sci. Math., 69(2003), 359-376.

[6] Bermúdez, T., Martinón, A., Müller, V., Noda, J., Perturbation of $m$-isometries by nilpotent operators, Abstr. Appl. Anal., 2(2014), 313-328.

[7] Berkani, M., On a class of quasi-Fredholm operators, Int. Eq. Op. Th., 34(1999), 244-249.

[8] Chō, M., Ota, S., Tanahashi, K., Uchiyama, M., Spectral properties of m-isometric operators, Functional Analysis, Application and Computation, 4(2012), no. 2, 33-39. 
[9] Chō, M., Ko, E., Lee, J., On m-complex symmetric operators, Mediterranean Journal of Mathematics, 13(2016), no. 4, 2025-2038.

[10] Chō, M., Ko, E., Lee, J., On m-complex symmetric operators, II, Mediterranean Journal of Mathematics, 13(2016), no. 5, 3255-3264.

[11] Colojoara, I., Foias, C., Theory of generalized spectral operators, Gordon and Breach, New York, 1968.

[12] Eschmeier, J., Invariant subspaces for operators with Bishop's property ( $\beta$ ) and thick spectrum, J. Funct. Anal., 94(1990), 196-222.

[13] Garcia, S.R., Aluthge transforms of complex symmetric operators and applications, Int. Eq. Op. Th., 60(2008), 357-367.

[14] Garcia, S.R., Putinar, M., Complex symmetric operators and applications, Trans. Amer. Math. Soc., 358(2006), 1285-1315.

[15] Garcia, S.R., Putinar, M., Complex symmetric operators and applications, II, Trans. Amer. Math. Soc., 359(2007), 3913-3931.

[16] Garcia, S.R., Wogen, W.R., Some new classes of complex symmetric operators, Trans. Amer. Math. Soc., 362(2010), 6065-6077.

[17] Halmos, P.R., A Hilbert space problem book, Springer-Verlag, Berlin - Heidelberg - New York, 1980.

[18] Helton, J.W., Operators with a representation as multiplication by $x$ on a Sobolev space, Colloquia Math. Soc. Janos Bolyai, 5, Hilbert Space Operators, Tihany, Hungary, 1970, 279-287.

[19] Jung, S., Ko, E., Lee, M., Lee, J., On local spectral properties of complex symmetric operators, J. Math. Anal. Appl., 379(2011), 325-333.

[20] Laursen, K., Neumann, M., An introduction to local spectral theory, Clarendon Press, Oxford, 2000.

[21] McCullough, S., Rodman, L., Hereditary classes of operators and matrices, Amer. Math. Monthly, 104(1997), no. 5, 415-430.

Muneo Chō

Department of Mathematics

Kanagawa University

Hiratsuka 259-1293, Japan

e-mail: chiyom01@kanagawa-u.ac.jp

Eungil Ko

Department of Mathematics

Ewha Womans University

Seoul 120-750, Korea

e-mail: eiko@ewha.ac.kr

Ji Eun Lee

Department of Mathematics and Statistics

Sejong University

Seoul 143-747, Korea

e-mail: jieun7@ewhain.net; jieunlee7@sejong.ac.kr 